\title{
Globular clusters and their contribution to the formation of the Galactic halo
}

\author{
Eugenio Carretta \\ INAF-Osservatorio Astronomico di Bologna, via Ranzani 1, I-40127 Bologna, Italy \\ email: eugenio.carretta@oabo.inaf.it
}

\begin{abstract}
This is a "biased" review because I will show recent evidence on the contribution of globular clusters (GCs) to the halo of our Galaxy seen through the lens of the new paradigm of multiple populations in GCs. I will show a few examples where the chemistry of multiple populations helps to answer hot questions including whether and how much GCs did contribute to the halo population, if we have evidence of the GCs-halo link, what are the strengths and weak points concerning this contribution.
\end{abstract}

Keywords. stars: abundances, stars: Population II, Galaxy: abundances, (Galaxy:) globular clusters: general, Galaxy: halo

\section{Introduction}

We obviously know that GCs $d$ o contribute to the Galactic halo, there are about 100 "smoking guns", i.e. the GCs currently observed in the Milky Way (MW) halo. Furthermore, large surveys like SDSS, Pan-STARRS, Dark Energy Survey, public ESO surveys such as VVV and ATLAS routinely discover new systems in the grey zone between globular clusters and dwarf galaxies, sometimes with controversial classification, see e.g. the case of Crater/Laevens1 considered to be a dwarf galaxy (Bonifacio et al. 2015) or a GC (Kirby et al. 2015a).

Mass loss is expected for GCs in particular at early phases, during the violent relaxation following gas expulsion (see e.g. Lynden-Bell 1967, Baumgardt et al. 2008) but GCs are dynamically evolved systems, hence mass loss is predicted during all their lifetime. It is then possible that many clusters dissolved, and what we see now are the smaller survivors of a potentially much larger population.

Early (e.g. Ibata et al. 1994, Fusi Pecci et al. 1995) and more recent studies (Forbes and Bridges 2010, Leaman et al. 2013) on the accretion history focussed on dwarf galaxies and entire clusters or entire systems of GCs, not on the direct contribution from the clusters themselves. A possible reason is summarized in Fig. 1: the chemical tagging made with $\alpha$-elements may distinguish between clusters and dwarf galaxies, but usually GC stars are superimposed to several Galactic components, formed in situ, accreted or even kicked out (see Sheffield et al. 2012). Clearly we need another diagnostic.

\section{The Na-O anticorrelation}

One of the best tools for chemical tagging of GC stars is the Na-O anticorrelation, which represents the unique DNA of GCs (see Fig. 2 which summarizes the results of our FLAMES survey with $\sim 2500$ red giant stars in 25 GCs, see Carretta 2015 for references). The huge spreads in $\mathrm{Na}$ and $\mathrm{O}$ tell us that GCs are not simple stellar populations (by definition, coeval stars with the same initial chemical composition) because GC stars have very different $\mathrm{Na}$, $\mathrm{O}$ contents. Hence, their stars are not strictly coeval: huge chemical 


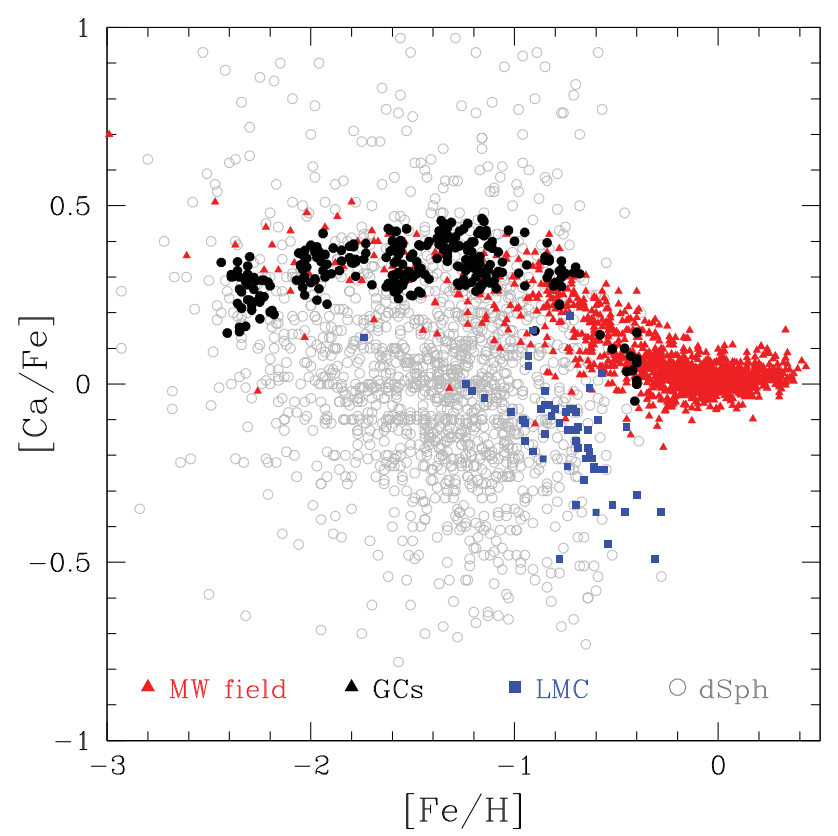

Figure 1. $[\mathrm{Ca} / \mathrm{Fe}]$ as a function of $[\mathrm{Fe} / \mathrm{H}]$ for several stellar populations. Stars in GCs (black filled circles) from Carretta et al. (2010a), Carretta et al. (2010c), Carretta et al. (2011), Carretta et al. (2013), Carretta et al. (2014a), Carretta et al. (2014b), Carretta et al. (2015); in dwarf galaxies (open grey circles) from Kirby et al. (2011); in LMC (filled squares) from Pompeia et al. (2008); and in the Milky Way (filled red triangles) from Adibekyan et al. (2012), Chen et al. (2000), Gratton et al. (2003), and Jonsell et al. (2005).

differences translate into tiny age differences, $10^{6}-10^{7}$ yrs, depending on the kind of polluter chosen to release enriched matter in the intracluster gas (see the review by Gratton et al. 2012 for details).

This chemistry is explained as the result of proton-capture reactions in H-burning at high temperature, enhancing or destroying different elements according to the temperature stratification (Denisenkov \& Denisenkova 1989).

The occurrence of this pattern is only seen in the high density GC environment, whereas in Galactic Pop. II field stars $\mathrm{O}$ and $\mathrm{Na}$ are basically untouched from the main sequence up to the giant branch (shaded box in Fig. 2, where the field sample is taken from Gratton et al. 2003) and Gratton et al. (2000).

The Na-O anticorrelation, discovered by the Lick-Texas group (e.g. Kraft 1994), joined other features like the anticorrelation of $\mathrm{C}$ and $\mathrm{N}$, observed even in unevolved stars, and of other elements along increasing Coulomb barrier like $\mathrm{Al}, \mathrm{Mg}$ and even $\mathrm{K}$ (Mucciarelli et al. 2012, Cohen \& Kirby 2012, Carretta 2014). In turn, these abundance variations have an impact on the photometric sequences in the color-magnitude diagrams due to molecular bands, mostly of CNO elements (Sbordone et al. 2011, Milone et al. 2012, Larsen et al. 2014a). For heavier species there is no direct photometric observable.

The anticorrelation of $\mathrm{Na}$ and $\mathrm{O}$ is the most notable of these features because it is observed in GCs spanning the whole mass range from the tiny Pal 5 (Smith et al. 2002) in dissolution phase to $\omega$ Cen (e.g. Johnson \& Pilachowski 2010), the likely remnant of a nucleated dwarf galaxy; it is observed in GCs of sure or suspected extragalactic origin (e.g. M 54, Carretta et al. 2010a) and in those showing a metallicity spread ( $\omega$ Cen, M 54). This signature does not discriminate between GCs formed in situ or 


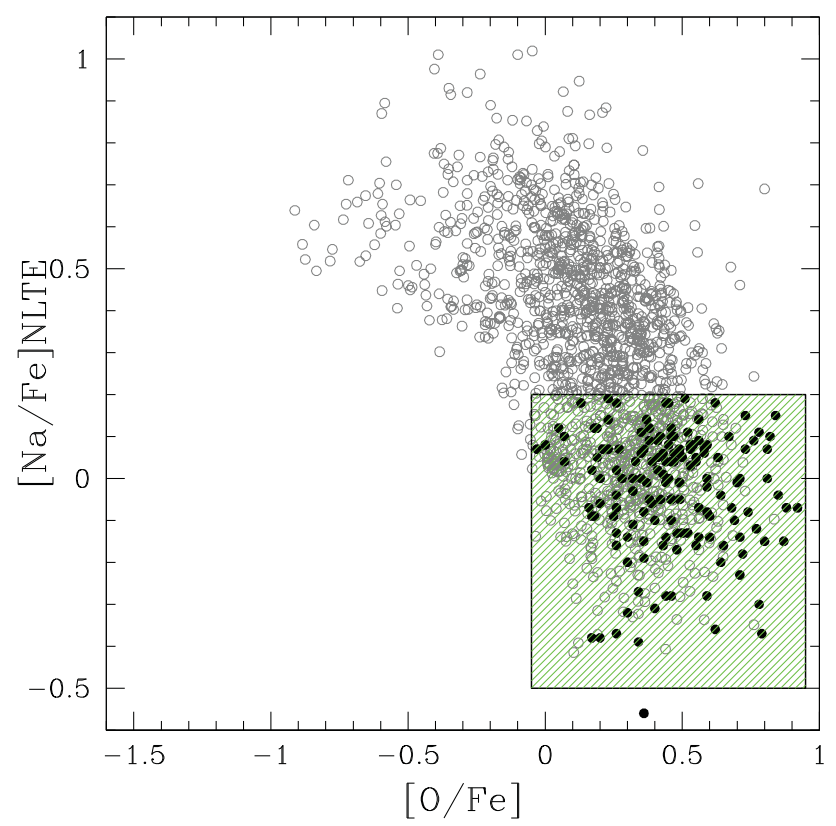

Figure 2. $[\mathrm{Na} / \mathrm{Fe}]$ as a function of $[\mathrm{O} / \mathrm{Fe}]$ for $\mathrm{GC}$ (empty circles, Carretta et al. 2009 and references in Fig.1) and field stars (filled circles, Gratton et al. 2003 and Gratton et al. 2000). The green shaded box encompasses the field stars.

likely accreted by our Galaxy (Leaman et al. 2013). In short, it is a widespread feature among GCs, likely related to the intrinsic mechanism of formation: we conclude that $a$ genuine old $G C$ is a system that formed in a short time at least two generations of stars with different content of proton-capture elements.

This signature allows us to put strong constraints on the initial masses of GCs. An almost constant fraction $(\sim 33 \%)$ of first generation $(\mathrm{FG})$ stars with primordial composition still resides in GCs (Carretta et al. 2009). However the bulk of present day GC stars is composed of second generation (SG) stars, with modified composition. Therefore, since SG stars are formed using the ejecta of only a fraction of FG stars, and the ejecta from the main candidate polluters are not enough (de Mink et al. 2009), we incur in the so called mass budget problem. This is solved with either a peculiar IMF or assuming that the precursors of GCs were much more massive than present-day GCs, losing about $90 \%$ of their stars and becoming good candidates as main contributors to the halo.

\section{Multiple populations in GCs: second generation stars in the halo}

A selective mass loss is expected because SG stars form more centrally concentrated in many models (e.g. D'Ercole et al. 2008, Decressin et al. 2007) and also the following dynamical evolution will push FG stars more easily outside tidal radius. Observations show that currently SG stars are usually more concentrated (e.g. Milone et al. 2012, Carretta 2015); however, after reaching full dynamical mixing, also SG stars with their unique chemical signature may be lost from GCs. These stars provide a clearcut probe of the contribution of GCs to the halo.

Carretta et al. (2010b) made a first attempt by comparing sodium abundances in field stars and SG stars in GCs. They found a small fraction (1.4\%) of stars with GC 
signature evaporated in the halo (the fraction is doubled by considering the current ratio of $\mathrm{FG} / \mathrm{SG}$ ).

A more systematic survey found a similar fraction of SG stars by looking for large $\mathrm{N}$ excess in metal-poor halo stars with spectra from the Sloan survey. After cleaning the sample for contamination by thick disk stars Martell et al. (2011) found 3\% of halo stars with SG composition, in agreement with serendipitous discoveries using $\mathrm{O}$ and $\mathrm{Na}$ (Ramirez et al. 2012), unless one of their two O-poor stars is found to be polluted by mass transfer from a companion AGB star, as suggested by the large abundance of barium and yttrium.

Finally, signatures of SG stars (Na, Al enhancements and O, Mg depletions) are more and more used to retrieve a cluster origin for streams (e.g. Sesar et al. 2015: Ophiuchus stream; Wylie-de Boer et al. 2012: Aquarius stream)

Using these signatures many studies (Carretta et al. 2010b, Vesperini et al. 2010, Schaerer \& Charbonnel 2011, Martell et al. 2011, Gratton et al. 2012) provide estimates for the fraction of halo stars originated in GCs. These range from a few percent, using only observations, up to almost $50 \%$ of the halo mass, if the initial mass of GCs is assumed to be many times larger than present-day values.

\section{Multiple populations in GCs: first generation stars in the halo}

We get important information also from the FG component, which is much more elusive, since FG stars share the same chemistry of field stars of similar metallicity. In the homogeneous set of $\mathrm{Na}$ abundance from Carretta (2013, empty triangles in Fig. 3 here) there are a few stars clearly below the general trend (filled triangles in Fig. 3): these are objects tagged as accreted stars (e.g. Gratton et al. 2003, Nissen \& Schuster 2010) and their abundances are clearly similar to those observed in dwarf satellites of Milky Way. Therefore, we may infer that a minority of halo stars has a composition similar to the dwarfs, but the bulk of halo stars looks like the FG component of GCs (red filled squares in Fig. 3).

This confirms the findings from $\alpha$-elements (Tolstoy et al. 2009) and carbon (Kirby et al. 2015b): the majority of field halo stars cannot have formed in present-day dSphs.

Another supporting evidence comes from the horizontal branch (HB) morphology. We know that the luminosity function of BHB stars is very different in GCs and in the halo (e.g. Kinman \& Allen 1996, Gratton et al. 2012). We further know that Na-rich stars are also more enriched in He. Carretta et al. (2007), Carretta et al. (2010b) found that the extension of the $\mathrm{Na}-\mathrm{O}$ anticorrelation is correlated to the maximum temperature reached at the bluest $\mathrm{HB}$ region (see Fig. 4) through the He abundance, increasing blueward. The maximum temperature on the HB of field BHB stars implies that they are not $\mathrm{He}$ enhanced. Since He-rich, Na-rich stars are rare in the field this suggests that the field BHB are related to the FG in GCs.

Additional evidence comes from binaries: Goodwin (2010) summarizes how the frequency and separation of binaries in different environments can be used to estimate the past density of their birth place. D'Orazi et al. (2010) and Lucatello et al. (2015) found that the binary fraction in large samples of GC stars is much lower among SG stars than among FG stars, with evidence of a formation in much denser environment, as predicted by theoretical models (Hong et al. 2015), and suggesting a common origin for the field stars and the FG populations. 


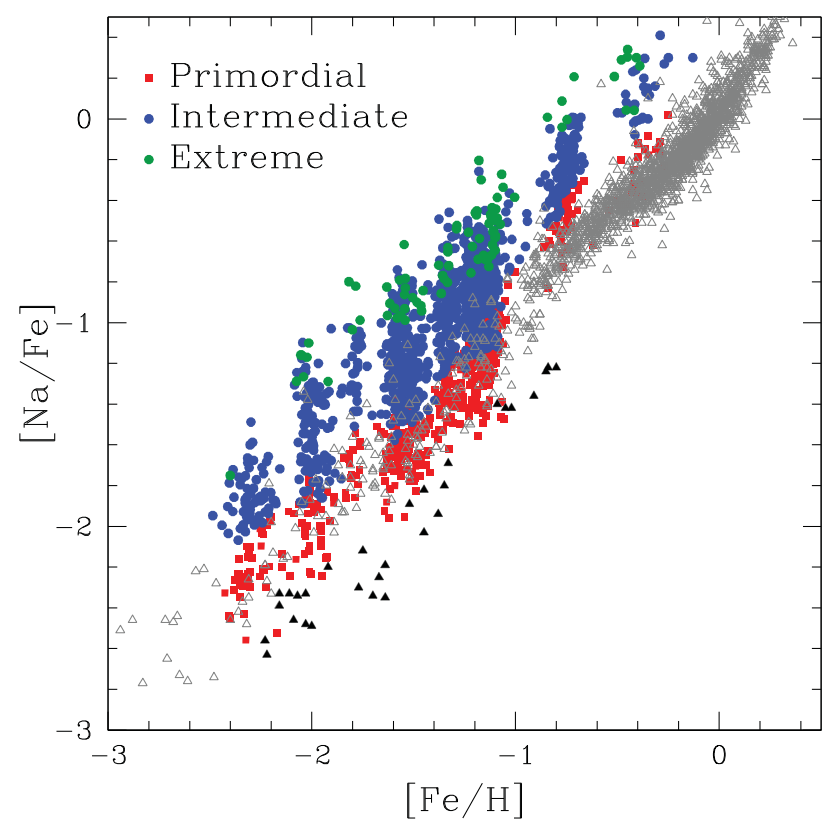

Figure 3. $[\mathrm{Na} / \mathrm{H}]$ as a function of $[\mathrm{Fe} / \mathrm{H}]$ for $\mathrm{GC}$ stars of different generations (filled squares and circles) and field stars (triangles). Filled triangles are the accreted field component.

\section{Challenges and conclusions}

The basic assumptions for a large contribution of GC stars to the halo (initial masses much larger, with selective loss of FG stars) must confront with two major challenges.

Evidence is accumulating that in dwarf galaxies GCs cannot have been initially more than 4-5 times today's mass, as their present-day masses account for about $25 \%$ of the galaxy mass in metal poor stars (Larsen et al. 2012, Larsen et al. 2014b, Tudorica et al. 2015). However, GCs like those in the Fornax dSph show normal evidence of multiple populations (Larsen et al. 2014a).

Furthermore, simulations of mass loss from gas expulsion (Khalaj \& Baumgardt 2015) predict a strong anticorrelation between the fraction of SG stars and the cluster mass, that is not observed. Moreover, no variation of the fraction of SG stars as a function of cluster mass or Galactocentric distance or metallicity is observed (Bastian \& Lardo 2015), at odds with what expected by mechanisms of mass loss.

The above points directly concern the multiple population scenario. However, independently from this scenario, larger birth cluster masses and large amount of mass loss are predicted, providing a mass comparable with the halo total mass (e.g. Marks \& Kroupa 2010, Fall \& Zhang 2001). In particular, primordial residual-gas expulsion and infant mortality may account for the bulk of the halo with FG signature (Baumgardt et al. 2008).

In summary, we have plenty of evidence for a relevant contribution of GCs to the halo: streams likely originated from GCs (from their size or chemical tagging, see also Grillmair, this volume), tidal tails, direct or indirect evidence from chemistry, HB stars and binary fraction. Considering that a good third of the halo seems due to the so-called "big four" accretion events (Sagittarius, Hercules-Aquila, GASS, Virgo Cloud; see Belokurov 2013), a large contribution of GCs to the general assembly of the Galactic halo seems to be a viable option. 


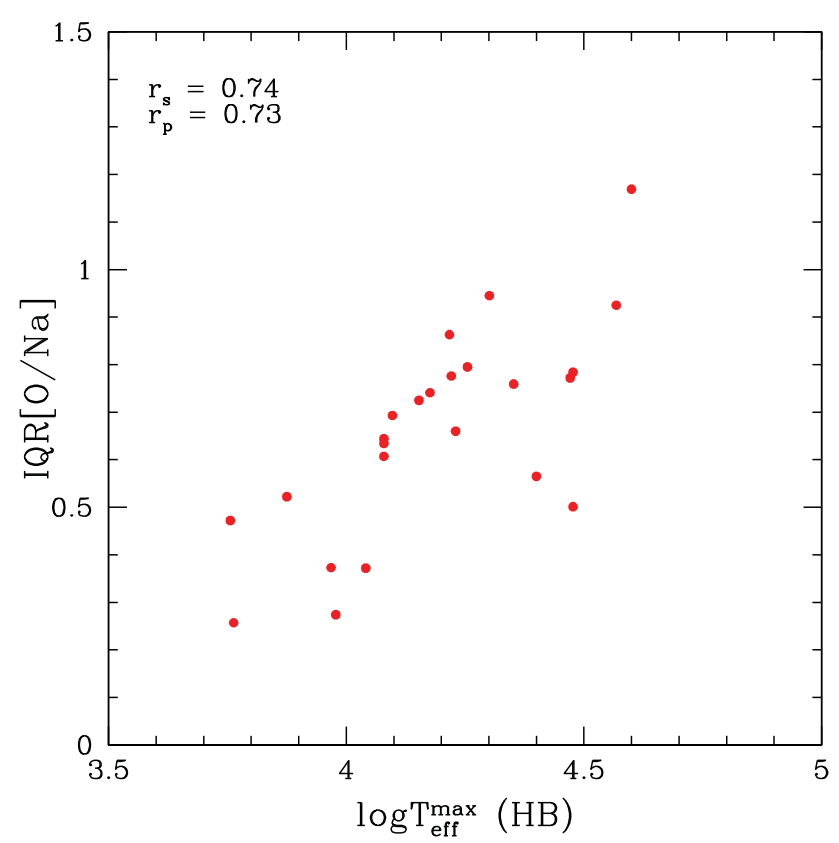

Figure 4. The extension of the $\mathrm{Na}-\mathrm{O}$ anticorrelation (measured by the interquartile range $\mathrm{IQR}[\mathrm{O} / \mathrm{Na}]$ as a function of the maximum temperature along the HB for GCs in the FLAMES survey (Carretta et al. 2007, Carretta et al. 2010b).

\section{References}

Adibekyan, V. Zh, Sousa, S. G., Santos, N. C., Delgado Mena, E., Gonzales Hernandez, J. I., Israelian, G., Mayor, M., \& Khachatryan, G. 2012, A\&SA, 545, A32

Bastian, N. \& Lardo, C. 2015, MNRAS, 453, 357

Baumgardt, H., Kroupa, P., \& Parmentier, G. 2008, MNRAS, 384, 1231

Belokurov, V. 2013, New AR, 57, 100

Bonifacio, P., Caffau, E., Zaggia, S., Franøis, P., Sbordone, L., Andrievsky, S. M., \& Korotin, S. A. $2015, A \& A, 579$, L6

Carretta, E. 2013, A\& $A, 557$, A128

Carretta, E. 2014, ApJ, 795, L28

Carretta, E. 2015, ApJ, 810, 148

Carretta, E., Recio-Blanco, A., Gratton, R. G., Piotto, G., \& Bragaglia, A. 2007, ApJ, 671, L125

Carretta, E., Bragaglia, A., Gratton, R. G., et al. 2009, A\&A, 505, 117

Carretta, E., Bragaglia, A., Gratton, R. G., et al. 2010a, A\&A, 520, 95

Carretta, E., Bragaglia, A., Gratton, R. G., Recio-Blanco, A., Lucatello, S., D'Orazi, V., \& Cassisi, S. 2010b, A\&A, 516, 55

Carretta, E., Bragaglia, A., Gratton, R., Lucatello, S., Bellazzini, M., \& D’Orazi, V. 2010c, ApJ, $712, \mathrm{~L} 21$

Carretta, E., Lucatello, S., Gratton, R. G., Bragaglia, A., \& D’Orazi, V. 2011, A\&A, 533, 69

Carretta, E., Bragaglia, A., Gratton, R. G. et al. 2013, A\& A, 557, A138

Carretta, E., Bragaglia, A., Gratton, R. G., D’Orazi, V., Lucatello, S., \& Sollima, A. 2014a, $A \mathscr{E} A, 561, \mathrm{~A} 87$

Carretta, E., Bragaglia, A., Gratton, R. G. et al. 2014b, A\&SA, 564, A60

Carretta, E., Bragaglia, A., Gratton, R. G. et al. 2015, A\& A, 578, A116

Chen, Y. Q., Nissen, P. E., Zhao, G., Zhang, H. W., \& Benoni, T. 2000, A\&AS, 141, 491

Cohen, J. G. \& Kirby, E. N. 2012, ApJ, 760, 86

Decressin, T., Meynet, G., Charbonnel C. Prantzos, N., \& Ekstrom, S. 2007, A\& $A, 464,1029$

de Mink, S. E., Pols, O. R., Langer, N., \& Izzard, R. G. 2009, A\&AA, 507, L1 
Denisenkov, P. A. \& Denisenkova, S. N. 1989, A.Tsir., 1538, 11

D'Ercole, A., Vesperini, E., D'Antona, F., McMillan, S. L. W., \& Recchi, S. 2008, MNRAS, 391, 825

D’Orazi, V., Gratton, R. G., Lucatello, S., Carretta, E., Bragaglia, A., \& Marino, A. F. 2010, ApJ, 719, L213

Fall, S. M. \& Zhang, Q. 2001, ApJ, 561, 751

Forbes, D. A. \& Bridges, T. 2010, MNRAS, 404, 1203

Fusi Pecci, F., Bellazzini, M., \& Cacciari, C., Ferraro F. R. 1995, AJ, 110, 1664

Goodwin, S. P. 2010, RSPTA, 368, 851

Gratton, R. G., Sneden, C., Carretta, E., \& Bragaglia, A. 2000, A\& A, 354, 169

Gratton, R. G., Carretta, E., Claudi, R., Lucatello, S., \& Barbieri, M. 2003, A\&A, 404, 187

Gratton, R. G., Carretta, E., \& Bragaglia, A. 2012, A\&AR, 20, 50

Hong, J., Vesperini, E., Sollima, A., McMillan, S. L. W., D’Antona, F., \& D'Ercole, A. 2015, MNRAS, 449, 629

Ibata, R. A., Irwin, M. J., \& Gilmore, G. 1994, Nature, 370, 194

Johnson, C. I. \& Pilachowski, C. A. 2010, ApJ, 722, 1373

Jonsell, K., Edvardsson, B., Gustafsson, B., Magain, P., Nissen, P. E., \& Asplund, M. 2005, $A \mathscr{E A}, 440,321$

Khalaj, P. \& Baumgardt, H. 2015, MNRAS, 452, 924

Kinman, T. D. \& Allen, C. 1996, ASP-CS, 92, 36

Kirby, E. N., Cohen, J. G., Smith, G. H., Majewski, S. R., Sohn, S. T., \& Guhathakurta, P. 2011, ApJ, 727, 79

Kirby, E. N., Simon, J. D., \& Cohen, J. G. 2015, ApJ, 810, 56

Kirby, E. N., Guo, M., Zhang, A. J., et al. 2015, ApJ, 801. 125

Kraft, R. P. 1994, PASP, 106, 553

Larsen, S. S., Strader, J., \& Brodie, J. P. 2012, A\&SA, 544, L14

Larsen, S. S., Brodie, J. P., Forbes, D. A., \& Strader, J. 2014a, AE $A, 565$, A98

Larsen, S. S., Brodie, J. P., Grundahl, F., \& Strader, J. 2014b, ApJ, 797, 15

Leaman, R., VandenBerg, D. A., \& Mendel, J. T. 2013, MNRAS, 436, 122

Lucatello, S., Sollima, A., Gratton, R. G., Vesperini, E., D’Orazi, V., Carretta, E., \& Bragaglia, A. $2015, A \& A$, in press, arXiv: 1509.05014

Lynden-Bell, D. 1967, MNRAS, 136, 101

Marks, M. \& Kroupa, P. 2010, MNRAS, 406, 2000

Martell, S. L., Smolinski, J. P., Beers, T. C., \& Grebel, E. K. 2011, A\&\&A, 534, 136

Milone, A., Piotto, G., Bedin, L. et al. 2012, ApJ, 744, 58

Mucciarelli, A., Bellazzini, M., Ibata, R., Merle, T., Chapman, S. C., Dalessandro, E., \& Sollima, A. $2012, M N R A S, 426,2889$

Nissen, P. E. \& Schuster, W. J. 2010, A\&A, 511, L10

Pompéia, L., Hill, V., Spite, M., Cole, A. et al. 2008 2008, A\& A, 480, 379

Ramírez, I., Meléndez, J., \& Chanamé, J. 2012, ApJ, 757, 164

Sbordone, L., Salaris, M., Weiss, A., \& Cassisi, S. 2011, A\&JA, 534, A9

Schaerer, D. \& Charbonnel, C. 2011, MNRAS, 413, 2297

Sesar, B., Bovy, J., Bernard, E. J. et al. 2015, ApJ, 809, 59

Sheffield, A. A., Majewski, S. R., Johnston, K. V. et al. 2012, ApJ, 761, 161

Smith, G. H., Sneden, C., \& Kraft, R. P. 2002, AJ, 123, 1502

Tolstoy, E., Hill, V., \& Tosi, M. 2009, ARA $6 A$, 47, 371

Tudorica, A., Georgiev, I. Y., \& Chies-Santos, A. L. 2015, A\&A, 581, 84

Vesperini, E., McMillan, S. L. W., D'Antona, F., \& D'Ercole, A. 2010, ApJ, 718, 112

Wylie-de Boer, E., Freeman, K., williams, M., Steinmetz, M., Munari, U., \& Keller, S. 2012, ApJ, 755, 35 